\title{
Source Coding for a Multihop Network*
}

\author{
Wei-Hsin Gu and Michelle Effros \\ California Institute of Technology
}

We bound the rate-distortion region for a four-node network. The results are the first known expansion of rate-distortion theory from single-hop networks (every source has a direct connection to each of its destinations), to multihop networks, which allow intermediate nodes.

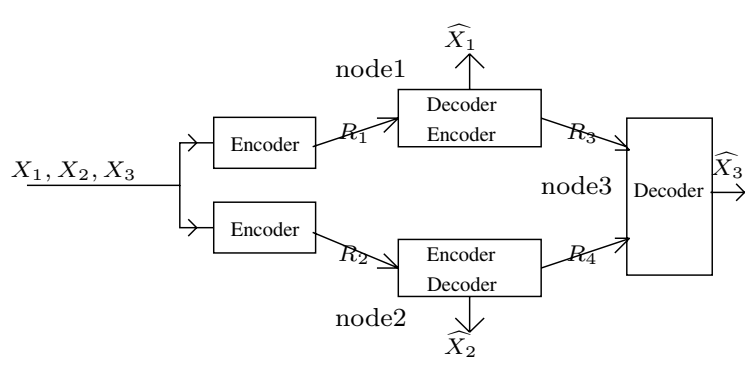

Figure 1: The diamond network.

Multihop networks exhibit a variety of characteristics absent from prior single-hop networks: a single source description may take multiple paths to its destination; multiple source descriptions may share a single link en route to different destinations; and intermediate nodes may process incoming descriptions and send partial descriptions on to subsequent nodes in the network. While single-hop network source coding solutions may be applied in multihop networks, such applications require explicit rate allocation for each sourcedestination pair, and the resulting solutions may be suboptimal. We therefore tackle the multihop network source coding problem directly. We use the diamond network illustrated in Figure 1, since it exhibits many of the described features.

Consider the diamond network from Figure 1. Vectors $\left(X_{1}, X_{2}, X_{3}\right)$ are drawn i.i.d. from probability mass function $p\left(x_{1}, x_{2}, x_{3}\right)$ on finite alphabet $\mathcal{X}_{1} \times \mathcal{X}_{2} \times \mathcal{X}_{3}$. For each $j \in\{1,2,3\}$, node $j$ reconstructs source $X_{j}$ with expected distortion $D_{j}$.

Theorem If $\left(R_{1}, R_{2}, R_{3}, R_{4}, D_{1}, D_{2}, D_{3}\right)$ is achievable for the diamond network then

$$
\begin{aligned}
& R_{i} \geq R_{X_{i} \mid U_{i}}\left(D_{i}\right)+I\left(X_{1}, X_{2}, X_{3} ; U_{i}\right), \quad i \in\{1,2\} \quad R_{X_{3} \mid U_{1}, U_{2}}\left(D_{3}\right)=0 \\
& R_{i} \geq I\left(X_{1}, X_{2}, X_{3} ; U_{i-2}\right), \quad i \in\{3,4\}
\end{aligned}
$$

for auxiliary random variables $U_{1}$ and $U_{2}$ defined by conditional probability mass function $p\left(U_{1}, U_{2} \mid X_{1}, X_{2}, X_{3}\right)$ on finite alphabets $\mathcal{U}_{1} \times \mathcal{U}_{2}$. On the other hand, $\left(R_{1}, R_{2}, R_{3}\right.$ , $\left.R_{4}, D_{1}, D_{2}, D_{3}\right)$ is achievable if it satisfies (1) with strict inequalities and $U_{1} \rightarrow$ $\left(X_{1}, X_{2}, X_{3}\right) \rightarrow U_{2}$ forms a Markov chain.

${ }^{*}$ This material is based upon work partially supported by NSF Grant No. CCR-0220039 and Caltech's Lee Center for Advanced Networking. 